JOURNAL OF THEORETICAL

AND APPLIED MECHANICS

58, 1, pp. 233-245, Warsaw 2020

DOI: $10.15632 /$ jtam-pl $/ 115550$

\title{
IDENTIFICATION OF THE PARAMETERS OF A VEHICLE CRASHING INTO A ROUND PILLAR
}

\author{
Robert Kostek, Piotr Aleksandrowicz \\ University of Science and Technology in Bydgoszcz, Poland \\ e-mail: robertkostek@o2.pl; piotr.aleksandrowicz@utp.edu.pl
}

\begin{abstract}
This article presents a study on reconstruction of a crash of a passenger car - Opel Mokka, into a pillar. Computer simulations were performed with software V-SIM4, both for default data and data identified from the crash test. The crash test was performed by AUTOBILD and DEKRA. The frontal collision with a pillar is not a standard crash test recommended by the Directives of Communauté Économique Européenne (CEE), even though this type of collision poses a serious threat to the safety of vehicle users. The threat comes from the large penetration of the vehicle body through the pillar. These accidents are difficult to reconstruct with the programs applied by expert witnesses, because they require a lot of experience and changes in many parameters. Identification of these parameters is critical in this case. Values of the parameters were identified from recorded images. The obtained results of simulation show strong sensitivity of the accident course to the position of the force application point, which acts between the pillar and the vehicle. Also, the key factors are: contact parameters, identification of the initial conditions, sensitivity of the course of the accident to the adopted values of the parameters, and knowledge of the limitations of any software. Many expert witnesses do not even realise that their results of simulations, based on default values, are faulty. The process of obtaining an agreement between the simulation and experimental results is a time-consuming iteration process. This process is described in this article, which is address to expert witnesses and researchers; moreover, a direction for development of software was suggested.
\end{abstract}

Keywords: crash simulation, collision with a pillar, V-SIM

\section{Introduction}

In 2017, the police in Poland reported 1667 vehicles crashing into trees, with a total loss of life of 376 people. Thus, the number of fatalities for every 1000 accidents is 226 . The presented data show that such collisions result in one of the most severe consequences among all kinds of traffic accidents (http://www.krbrd.gov.pl/pl/test.html). Usually, a judge retains an independent expert who assists the court to draw correct inferences in decision-making. Expert witnesses analyse evidences and make simulations in a program. Discussed results of simulations are presented as a report which results in legal and economic consequences for the parties in the proceedings; therefore these reports should be carefully worked out. Crashes into poles and trees are not a standard crash tests (Radu and Cofaru, 2015); thus, it makes it very difficult to obtain reliable data for computer simulations. In the literature known to the authors, the results obtained with V-SIM4 were not validated against vehicle collision with a pillar, even though the program is commonly used by expert witnesses in Poland. Consequently, there is a need to describe the problems related to the credibility of simulation results, and the sensitivity of the course of such a collision to initial conditions. This issue is important for proper modelling of accidents and have practical implications in judicial decisions. The knowledge about the sources 
of simulation errors and the sensitivity of simulation results to input data is not common among expert witnesses; thus the simulation results for default settings are often accepted without any reservations.

The selection of a program for crash simulation depends on the requirements. Collisions can be simulated in Multi Body System (MBS) based software or Finite Element Method (FEM). The research institutions usually use FEM software, which provides an opportunity to perform precise calculations; nevertheless, FEM simulations are sporadic in the practice of road accident reconstruction as they require a long CPU time $(10 \mathrm{~h})$, and have to enter a lot of material and geometric data. A number of research articles present FEM simulations; for example Abdel-Nasser (2013) simulated a car crash into a street lamp pillar in Abaqus. The author observed that the crash resulted in a smaller car body deformation for a less stiff pillar comparing with a stiffer pillar. Whereas another study (Elmarakbi et al., 2006) demonstrated the effect of column base connections on the course of the crash and safety of the vehicle users. It is related to the shearing of the anchor bolts and a smaller car body deformation. Destruction of the anchor bolts is a separate issue which was reported by Stopel and Skibicki (2016). In another paper, Pawlak (2016) simulated in LS-DYNA deceleration of the car and its occupants during the crash into a pillar. It was found that the stiffness of a pillar and the type of soil around the pillar foundation affect the observed deceleration.

To shorten the CPU time, comparing to FEM simulation, one can apply modelling in the DyMesh convention. Then, the car body is modelled using a 3D triangle mesh, which is deformed during a collision. Nevertheless, modelling of acting forces is simplified as compared to FEM. The DyMesh convention provides an opportunity to simulate the frontal crash of the vehicle with a pillar, which was presented in (York and Day, 1999). This model of contact can be used in MBS programs, which are widely used in practice; it refers for example to V-SIM and PC-Crash (http://www.cyborgidea.com.pl; http://www.dsd.at). The MBS programs use the following contact models between vehicles and road barriers. The simplest contact detection method is based on the identification of common parts projections on a horizontal surface. It is also possible to apply a 3D model, then the common volume is detected. This approach is very similar to DyMesh. V-SIM4 offers both methods, which has been described in the paper by Aleksandrowicz (2017). The simplest contact characteristic assumes linear dependencies between the car body deformation and the contact force $F_{c}$. For that reason the term "car body stiffness" has been assumed for elastic and plastic deformations. Another article (Prochowski and Żuchowski, 2015) presented the effect of the engine unit and the elements being inside the vehicle engine compartment on the structure stiffness; which, in turn, affected the car body deformation. The presented results show that the stiffness of various car body areas differs and, additionally, the stiffness changes during collision - deformation. If during the crash the pillar crashes into the engine or side-member, then the stiffness is larger, and the car body deformation is smaller. However, if during the crash the pillar moves between the engine and the side-member, then the stiffness is lower, and the car body deformation is larger. This demonstrates that assuming an averaged stiffness for the entire car body is a simplification.

Next to the simulations, experiments were also performed. For experimental studies Ford Fiesta was used (Pawlus et al., 2011, 2013; Mnyazikwiye et al., 2013). The obtained results show that the Kelvin model is suitable for modelling a car crash into a pillar. Similar studies were described in another article (Ispas and Nastasoiu, 2017). The authors reported that for high speeds the car body penetration is large and the engine and gear box were moving towards the safety cage, which poses a serious threat to the safety of vehicle users.

Moreover, contact parameters were identified with V-SIM4 from crash tests with impact block (Kostek and Aleksandrowicz, 2017a). Then V-SIM4 was used to simulate crashes for default and identified data, which provides an opportunity to show the sensitivity of post impact movement 
to input data (Kostek and Aleksandrowicz, 2017b). The authors demonstrated that for the accurate simulations the default data were changed, otherwise applying default parameters can lead to significant errors. Summarising, the authors did not find validation of V-SIM for vehicle crash into the pillar, thus the problem is still present.

This article is divided into five Sections. The second Section presents the model of collision and a brief characteristic of the vehicle. The third Section compares experimental results of a crash test against results of simulation obtained for default data. Then the fourth Section provides simulation results obtained for the identified data compared against the experimental results. This shows the sensitivity of the course of collision to the adopted data. Finally, the most crucial observations are presented.

\section{Vehicle collision model in V-SIM4}

V-SIM4 is dedicated to simulation of traffic collision between vehicles and crash vehicles into road barriers. Two co-ordinate systems are employed. The first, the global system, describes position of vehicles, buildings and barriers. Its axes are marked as $x, y, z$, and its origin is determined by the program user. The second system is related to the simulated vehicle $x^{\prime}, y^{\prime}, z^{\prime}$. The origin of the second system is in the vehicle mass centre $C$, whose position is described by the vector $\mathbf{r}_{C}$. The axis $x^{\prime}$ is directed straight ahead, the axis $z^{\prime}$ is directed vertically upwards, whereas the axis $y^{\prime}$ is directed perpendicular to $x^{\prime} z^{\prime}$ and parallel to the ground (Fig. 1). The external forces acting on the vehicle are determined in the second system. The vehicle in V-SIM4 is modelled as a 16 degrees of freedom system. Six degrees of freedom (coordinates) describe the movement of the car body, whereas ten describe the position of the wheels. The resultant force acting on the vehicle is a sum of the contact forces acting between the car body and other objects, the gravitational force, the aerodynamic drag and the forces acting between the tyres and road. The force acting between the tire and road is determined by the tyre model "TM-Easy" as it well describes modern tyres. The program models an independent vehicle wheel suspension with progressive characteristics of springs and two damping coefficients - for compression and extension. Whereas, the steering system of the vehicle is modelled as ideal (Ackerman steering geometry, Rill, 2007). The program offers also the possibility to change the mass of passengers and luggage. In the program, one can also change the moments of inertia; however, they are constant during a collision, which is a simplification; because moments are usually reduced during a collision. In the studied case, the vehicle is shortened by about $0.81 \mathrm{~m}$, and so the moments of inertia are reduced, which is described in the fourth Section.

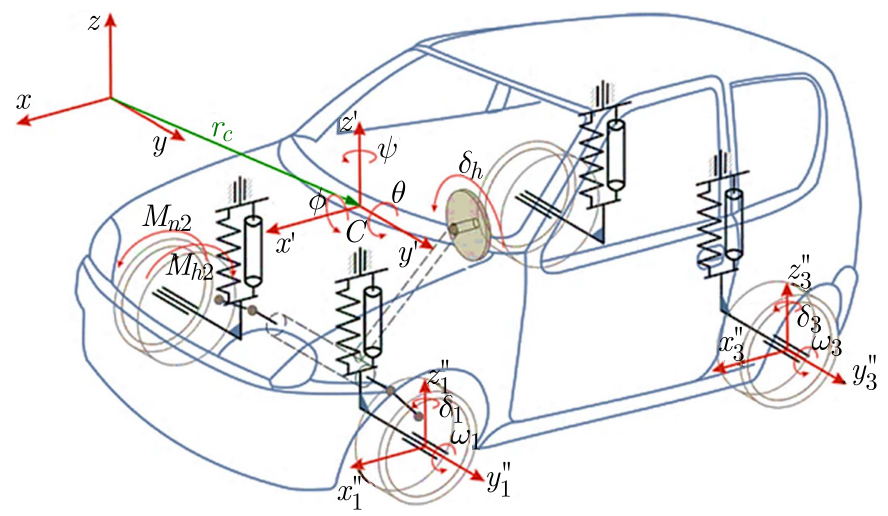

Fig. 1. Model of the vehicle with co-ordinate systems (http://www.cyborgidea.com.pl) 


\section{Results of simulation obtained for default data}

The masses of the Opel Mokka $(1425 \mathrm{~kg})$, the driver $(75 \mathrm{~kg})$, the passenger $(75 \mathrm{~kg})$ as well as the values of the moments of inertia (Table 1) were taken from the V-SIM4 database; whereas the mass of cargo $(100 \mathrm{~kg})$ and its position were taken from the crash test description (Rücker, 2016). The mass of apparatus was assumed $(30 \mathrm{~kg})$. It gives the real total mass of the vehicle being $1705 \mathrm{~kg}$. The height of the centre of mass of the vehicle and the height of the contact force application point were $0.59 \mathrm{~m}$ from the ground. Vectorial draws of the vehicle were taken from the databases Ratschbacher AutoView and Blueprints (https://www.autoview.at; https://www.theblueprints.com) (Fig. 2b).

Table 1. Default values of the parameters adopted to simulations

\begin{tabular}{|l|c|}
\hline \multicolumn{1}{|c|}{ Adopted parameters } & Default data \\
\hline \hline Adhesive friction coefficient & 0.90 \\
\hline Slip friction coefficient & 0.80 \\
\hline Rolling resistance coefficient & 0.015 \\
\hline Stiffness of car body for compression phase & $698 \mathrm{kN} / \mathrm{m}^{3}$ \\
\hline Stiffness of car body for restitution phase & $698 \mathrm{kN} / \mathrm{m}^{3}$ \\
\hline Restitution coefficient & 0.88 \\
\hline Friction coefficient between pillar and car & 1.87 \\
\hline Height of contact force application point & $0.590 \mathrm{~m}$ \\
\hline Lowering height of contact force application point $\Delta z^{\prime}$ & $0.0 \mathrm{~mm}$ \\
\hline Side displacement of contact force application point $\Delta y^{\prime}$ & $0.0 \mathrm{~mm}$ \\
\hline Time of front wheels blocking & - \\
\hline Moment of inertia $I x^{\prime}$ & $618 \mathrm{kgm}^{2}$ \\
\hline Moment of inertia $I y^{\prime}$ & $2598 \mathrm{kgm}^{2}$ \\
\hline Moment of inertia $I z^{\prime}$ & $2266 \mathrm{kgm}^{2}$ \\
\hline
\end{tabular}

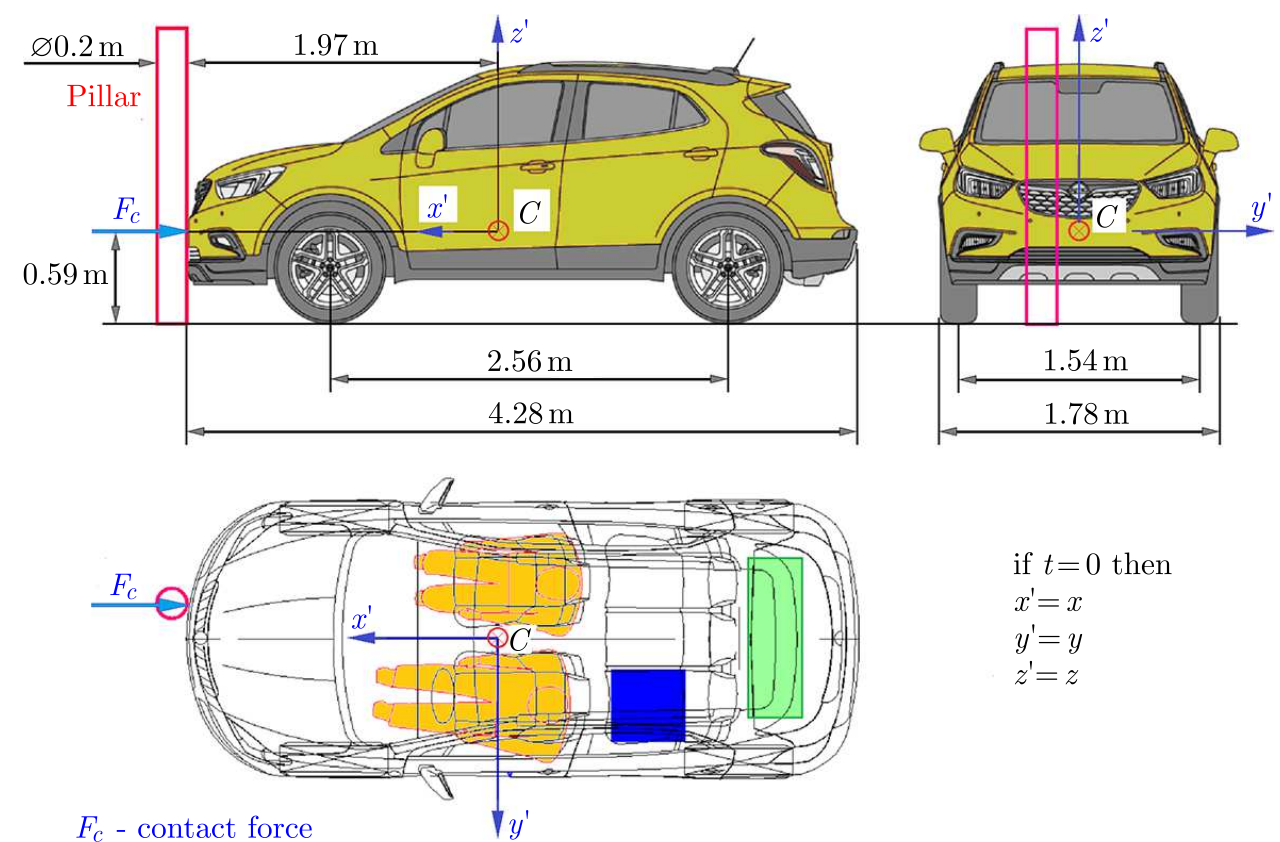

Fig. 2. Position of driver and passenger dummies in the vehicle (orange), cargo (green rectangle $100 \mathrm{~kg}$ ) and measurement apparatus (blue square $30 \mathrm{~kg}$ ) 
The crash test (https://www.youtube.com/watch?v=S4NFACR06Yc\&t=2s) was performed for the initial speed of $80 \mathrm{~km} / \mathrm{h}$. The vehicle crashed into a steel pillar $0.20 \mathrm{~m}$ in diameter (Fig. 3a). The collision was filmed from the top and from the side with high speed cameras (1000 fps), which made it possible to compare the simulation and experimental results. Time $t=0 \mathrm{~ms}$ was assumed for the moment of contact initiation. It was assumed that at $t=0 \mathrm{~ms}$ the axes of two coordinate systems $(x, y, z)$ and $\left(x^{\prime}, y^{\prime}, z^{\prime}\right)$ overlapped (Fig. 2). To compare the results, the images recorded from the top and pictures obtained from V-SIM4 were overlapped. The first stage of the reconstruction is to match the scale of the images. While scaling, edges, stripes, marks and geometrical data are crucial. This method is also applied to estimate vehicle body deformation. Then the rear window, rear door and roof are fitted to V-SIM drawing, because they are not significantly deformed. The same method is used in accident reconstruction. Having placed and scaled the drawing and photograph, it turned out that the upper camera was too close to the car, which resulted in a parallax error. The parallax error was $0.20 \mathrm{~m}$ for the upper edge of the windscreen, because a bumper was fitted in this case (Fig. 3a). To limit the error of measured displacement, the area near the pillar was matched. In practice, expert witnesses use recordings from CCTV (closed-circuit television) cameras, recorded at $25 \mathrm{fps}$ and low resolution, which makes image processing even more difficult. At the time instant $t=0$, the axis of symmetry of the pillar was $0.24 \mathrm{~m}$ from the plane of symmetry of the vehicle body (Fig. 3b).

(a)

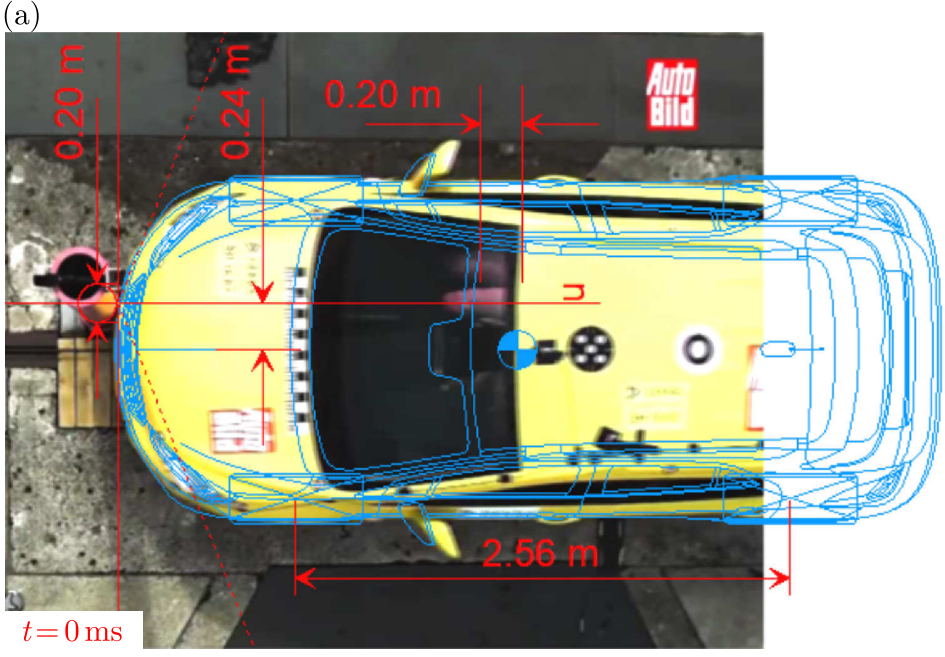

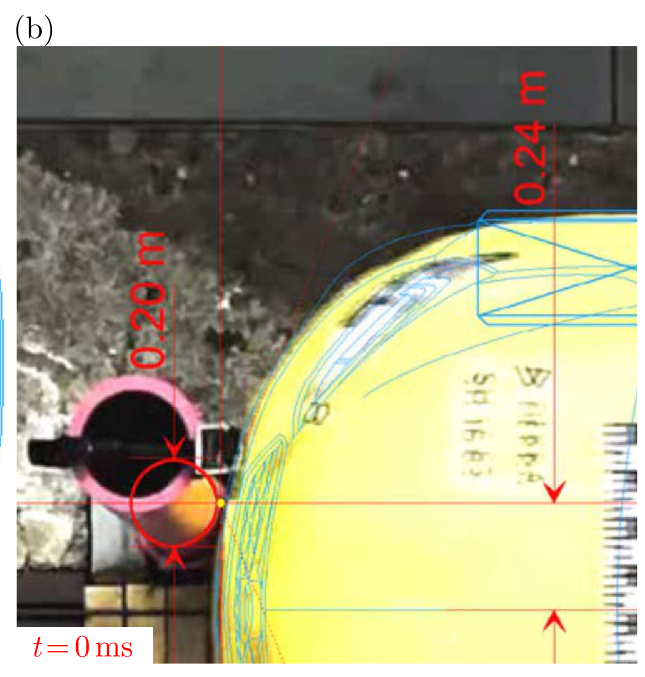

Fig. 3. Scaling image recorded by the camera and a top view of Opel Mokka for $t=0 \mathrm{~ms}$; measurement of the parallax error $(0.20 \mathrm{~m})$ and lateral displacement $(0.24 \mathrm{~m})$ (a) and enlarging the area of contact (b)

Having positioned the vehicle, simulation was conducted for the default data offered by the program (Table 1). Then the simulation results were compared against the images recorded from the top (Figs. 3 and 4). It was found that for the default parameters, a satisfactory agreement between the simulation and experimental results occurred only at the initial stage of the collision, namely from $t=0 \mathrm{~ms}$ to $t=60 \mathrm{~ms}$; when the vehicle body deformation was moderate (Fig. 4a,b). Next, with time, the position error increases and the reconstruction of the collision gets worse and worse. And, at $t=120 \mathrm{~ms}$, the ahead displacement of the modelled vehicle is clearly larger than that observed experimentally (Fig. 4c). Then the difference between the angles of rotation (yaw) increases. Finally, at $t=240 \mathrm{~ms}$, the difference between the yaw angles is $-33.4^{\circ}$, which leads to clear differences between the positions of the vehicles (Fig. 4d). The program does not reconstruct well the vehicle rotation. Summarising, for the default parameters the simulation results were definitely different than the course of the crash test, which is disturbing since the expert witnesses most often apply the default data, and so they receive the results as presented. 
Finding the reasons of described differences is crucial for reconstruction of accidents. The crash test analysis shows that during the collision the following phenomena occur:

- increase of side displacement of the vehicle body during the collision $(0.2 \mathrm{~m})$,

- vehicle shortening $(0.81 \mathrm{~m})$ and decreasing the moments of inertia $\left(680 \mathrm{kgm}^{2}\right)$,

- loss of contact of the rear wheels,

- pillar deflection.

The phenomena result in a different collision course as compared to the simulation results, and so they are modelled in the next Section.
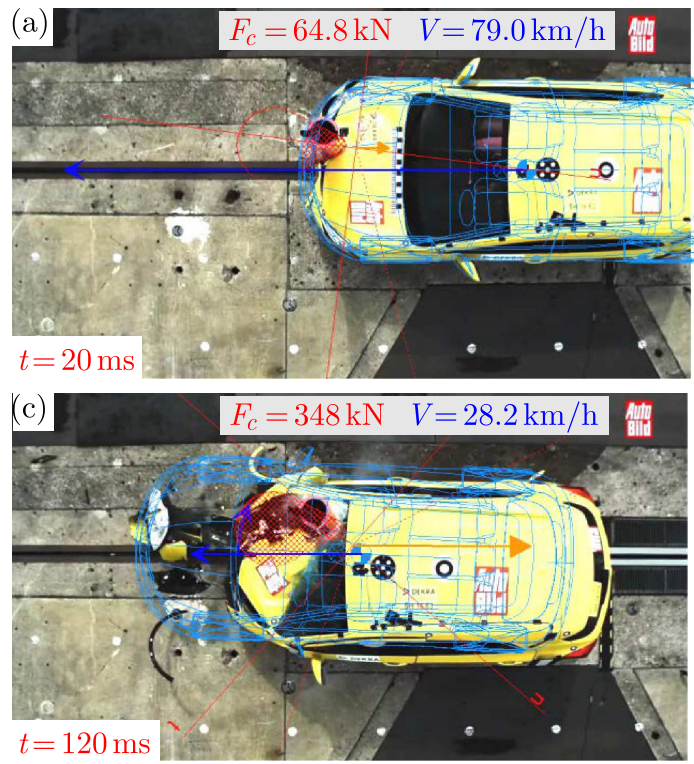
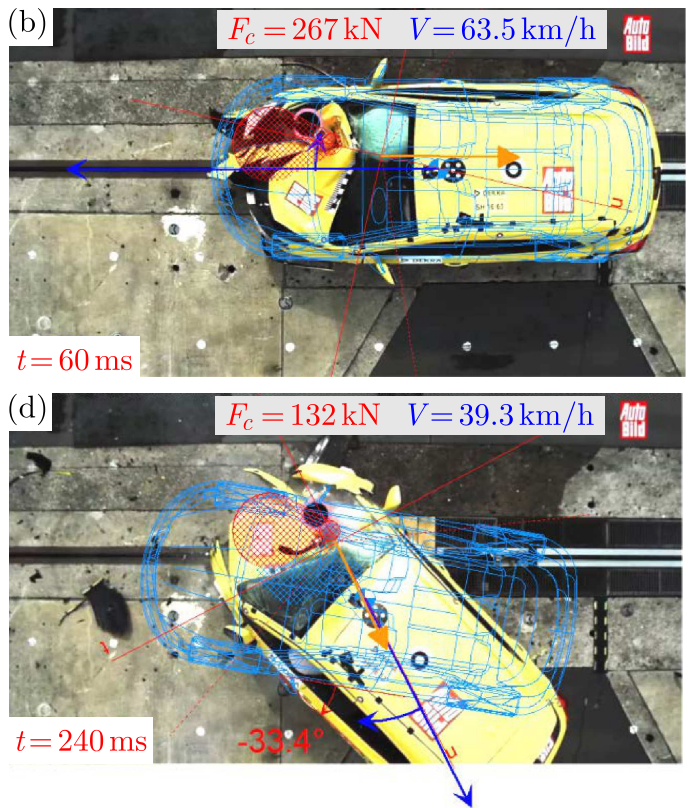

Fig. 4. Comparison of top views with the recorded images for consecutive times: (a) $t=20 \mathrm{ms,}$ (b) $t=60 \mathrm{~ms}$, (c) $t=120 \mathrm{~ms}$ and $9 \mathrm{~d}) t=240 \mathrm{~ms}$

\section{Results of simulation obtained for the identified parameters}

This Section describes how the collision parameters were changed in order to reduce the car body penetration by the pillar, to increase the yaw angle and, in turn, to get a satisfactory agreement. Stiffness for the compression phase was increased to reduce the vehicle body penetration. Immediately after the compression phase, the vehicle had small post-impact velocity, thus the restitution coefficient was reduced and the stiffness in the restitution phase was increased. The stiffness in the restitution phase is large.

The key factor, which resulted in a smaller yaw angle for "the default model" as compared with the real vehicle, is the increase of the side displacement of the vehicle during the collision. At $t=0 \mathrm{~ms}$, the side displacement is $0.24 \mathrm{~m}$ (Fig. 3), whereas at $t=136 \mathrm{~ms}$ it is almost twice more $0.44 \mathrm{~m}$, which is depicted in Fig. 5a. To verify this, a comparative measurement was executed for times $t=0 \mathrm{~ms}$ and $t=136 \mathrm{~ms}$. The measurement was based on the corner of the windscreen. The measured increase of the side displacement was $0.20 \mathrm{~m}$ (Fig. 5b); which finally gives the arm of the contact force being $0.44 \mathrm{~m}$, and so it is as much as it was measured before. The additional displacement appeared due to the contact between the pillar and engine unit. To reflect this, the side displacement of the contact force application point $\left(\Delta y^{\prime}=-0.20 \mathrm{~m}\right)$ was introduced. Finally, a longer arm results in a larger yaw moment $M_{z^{\prime}}$ and a larger yaw angle $\Psi$. 
Moreover, the vehicle shortening by $0.81 \mathrm{~m}$, displacement of some parts from the engine compartment to the passenger compartment, and cargo displacement from the boot into the back seats (Figs. 6a,c,d and 7b), decreased moments of inertia $I_{Y}$ and $I_{Z}$, and thus increased the yaw angle $\Psi$. The displacement of the engine unit with other parts $(250 \mathrm{~kg})$ and the cargo $(100 \mathrm{~kg})$ could reduce the moments of inertia by about $680 \mathrm{kgm}^{2}$, which was estimated from the following formula

$$
\Delta I_{Y}=\Delta I_{Z}=\left(R_{e 1}^{2} m_{e}+R_{l 1}^{2} m_{l}\right)-\left(R_{e 2}^{2} m_{e}+R_{l 2}^{2} m_{l}\right)
$$

where: $\Delta I_{Y}, \Delta I_{Z}$ - denote decreases of the moments of inertia $\left[\mathrm{kgm}^{2}\right], m_{e}=250 \mathrm{~kg}-$ represents mass of the engine together with nearby systems and parts of chassis, $R_{l e}=1.59 \mathrm{~m}$ is the distance between the centre of mass of the drive unit and the centre of mass of the vehicle prior to the crash, $R_{e 2}=0.90 \mathrm{~m}$ is the distance between the centre of mass of the drive unit and the centre of mass of the vehicle at $t=136 \mathrm{~ms}, m_{l}=100 \mathrm{~kg}$ - mass of cargo, $R_{l 1}=1.76 \mathrm{~m}$ is the distance between the centre of mass of the cargo and the centre of mass of the vehicle prior to the crash, $R_{l 2}=0.75 \mathrm{~m}$ is the distance between the centre of mass of the cargo and the centre of mass of the vehicle during the crash. The radii were estimated based on the photographs and drawings (Figs. 6a,c,d). To reflect this phenomenon, the moments of inertia $I_{Y}$ and $I_{Z}$ were reduced by $\Delta I_{Y}$ and $\Delta I_{Z}$. The real vehicle does not rotate, until large deformations take place; thus assuming smaller moments of inertia from the beginning is acceptable.
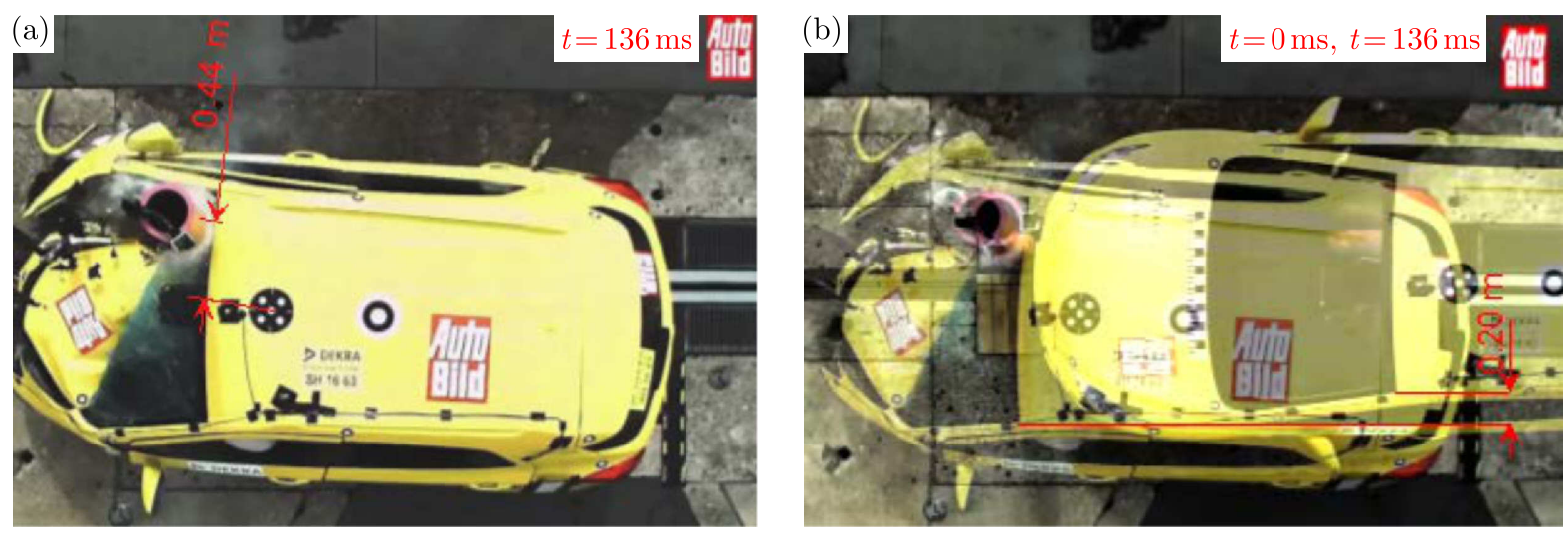

Fig. 5. Distance between the plane of symmetry of the vehicle and axis symmetry of the pillar $t=136 \mathrm{~ms}(\mathrm{a})$, increase of the lateral displacement from $t=0 \mathrm{~ms}$ to $t=136 \mathrm{~ms}(\mathrm{~b})$

The next phenomenon, which increases the yaw moment $M_{z}$ and yaw angle $\Psi$, is the loss of contact of the rear wheels. The experimental results show that the loss of contact begins in the time interval $140-159 \mathrm{~ms}$, and ends at $t=313 \mathrm{~ms}$; whereas for the modelled vehicle it begins in the time interval $100-160 \mathrm{~ms}$, and ends in time interval $320-520 \mathrm{~ms}$. This shows an agreement between the experimental and computational results. To reflect this phenomenon, the height of the contact force application point was reduced to $0.508 \mathrm{~m}$, which introduced the pitch moment $M_{y^{\prime}}$ and pitch angle $\Theta$ (Figs. 6b,d). Consequently, the vehicle rotated with a larger speed without significant friction $M_{z^{\prime}}$, when the rear wheels were off the ground (Figs. 10b,d). Now, the contact force acts slightly above the level of the floorpan, which reflects the resistance of the floorpan at the last compression phase. Interestingly, for the default settings, getting the rear wheels off the ground was not observed.

Besides, the pillar surface is smooth and is painted, which reduces the value of the friction coefficient. The smaller friction coefficient between the pillar and the car body slightly increases yaw angle. The assumed value corresponds to the friction pair steel to steel.

During the crash test, the steering system, suspension, drive systems and half-shaft are deformed. Consequently, the right front wheel breaks from $t=40 \mathrm{~ms}$ (Figs. $7 \mathrm{~b}$ and $9 \mathrm{c}$ ). Finally, 


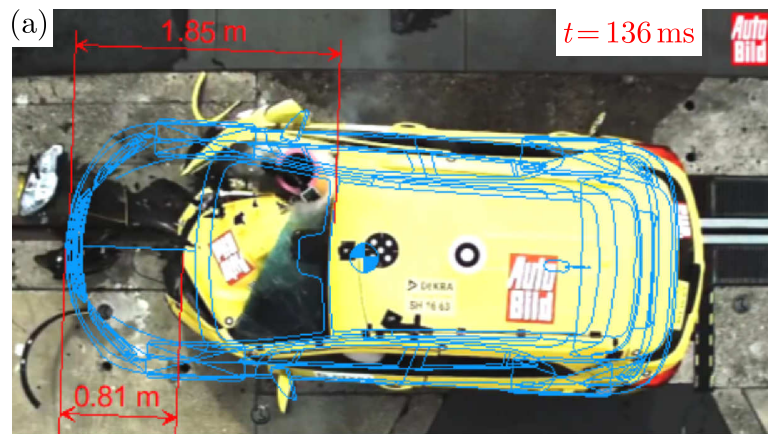

(b)
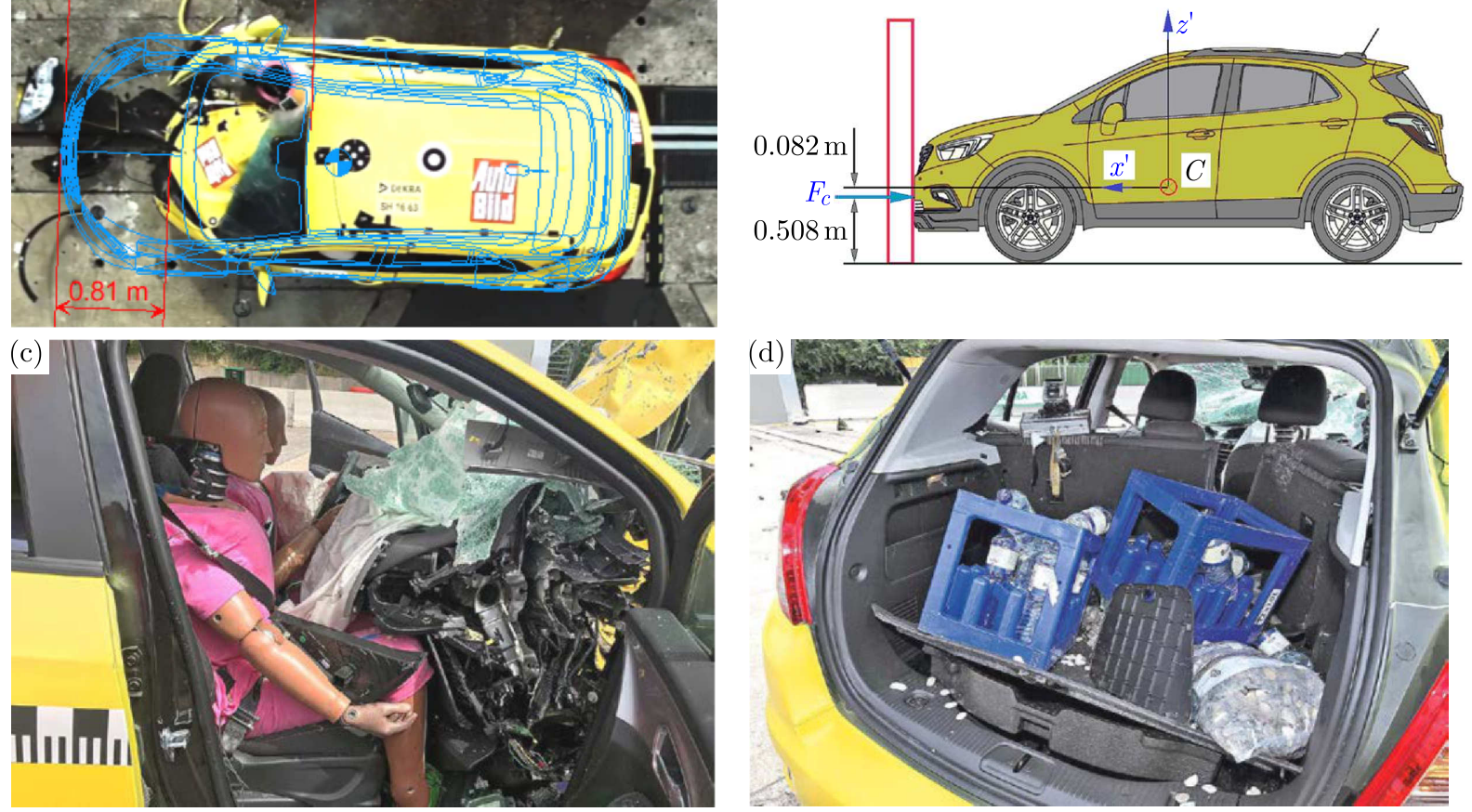

Fig. 6. Measured car body shortening $(0.81 \mathrm{~m})$ and penetration $(1.85 \mathrm{~m})$ (a), height of the contact force application point $(0.508 \mathrm{~m})(\mathrm{b})$, displacement of parts from the engine compartment deep into the passenger compartment (c), cargo after the crash (d), https://www.automagzz.com/so-we-crashed-a-5-star-car
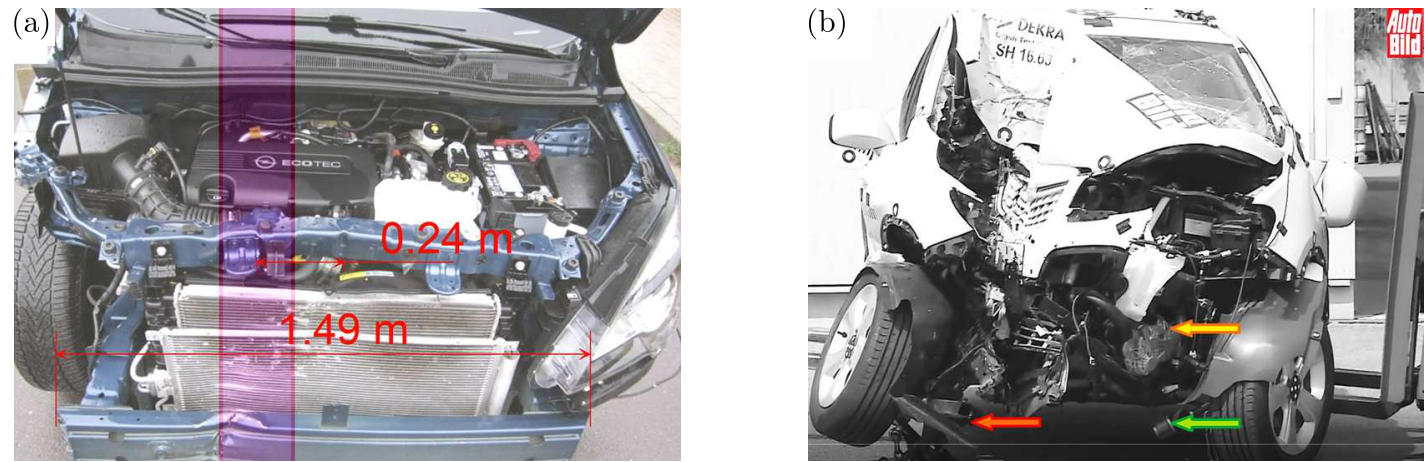

Fig. 7. Position of the pillar at $t=0 \mathrm{~ms}$ (a), the vehicle after the crash test (b), the red arrow marks the broken-off control arm while the green one - broken-off half-shaft, and the yellow one points to the turned engine

resistance to motion increases. To model this phenomenon, the front wheels were blocked from the time being $t=100 \mathrm{~ms}$. During the phases of compression and restitution, the braking force is not crucial because the contact force is over thirty times larger. Nevertheless, the braking force determines how far the vehicle drives away after the impact (post-impact motion).

Summarising, the introduced changes provide an opportunity to better describe this crash. It must be stressed that this program does not suggest introducing any modifications. In the literature known to the authors, these issues are not covered extensively; and thus they are described in this article.

Having introduced changes in many parameters, simulation was conducted, and the obtained results were compared against the experimental results. The presented results were described in a chronological order. At $t=0 \mathrm{~ms}$, the contact between the vehicle and pillar is initiated. 
Table 2. Values of parameters adopted to simulations

\begin{tabular}{|l|c|}
\hline \multicolumn{1}{|c|}{ Adopted parameters } & Identyfied data \\
\hline \hline Adhesive friction coefficient & 0.90 \\
\hline Slip friction coefficient & 0.80 \\
\hline Rolling resistance coefficient & 0.015 \\
\hline Stiffness of car body for compression phase & $1000 \mathrm{kN} / \mathrm{m}^{3}$ \\
\hline Stiffness of car body for restitution phase & $5000 \mathrm{kN} / \mathrm{m}^{3}$ \\
\hline Restitution coefficient & 0.07 \\
\hline Friction coefficient between pillar and car & 0.12 \\
\hline Height of contact force application point & $0.508 \mathrm{~m}$ \\
\hline Lowering height of contact force application point $\Delta z^{\prime}$ & $0.082 \mathrm{~m}$ \\
\hline Side displacement of contact force application point $\Delta y^{\prime}$ & $-0.20 \mathrm{~m}$ \\
\hline Time of front wheels blocking & $100 \mathrm{~ms}^{2}$ \\
\hline Moment of inertia $I x^{\prime}$ & $618 \mathrm{kgm}^{2}$ \\
\hline Moment of inertia $I y^{\prime}$ & $1918 \mathrm{kgm}^{2}$ \\
\hline Moment of inertia $I z^{\prime}$ & $1586 \mathrm{kgm}^{2}$ \\
\hline
\end{tabular}

For this time, a good agreement of the positions is obtained for the bumper (Fig. 9a). Then, at $t=20 \mathrm{~ms}$, the cross member marked in black and vehicle radiators are bent (Figs. 7a and 9b). At this time, the lower edges of the windscreen overlap. After that, at $t=40 \mathrm{~ms}$ the control arm is deformed. Additionally, the engine unit collides with the pillar. The collision results in a considerable deflection of the pillar towards the $x$ axis $(0.17 \mathrm{~m}$, Fig. 8). At this time an agreement of the position of the lower corners of the windscreen is obtained (Fig. 9c).

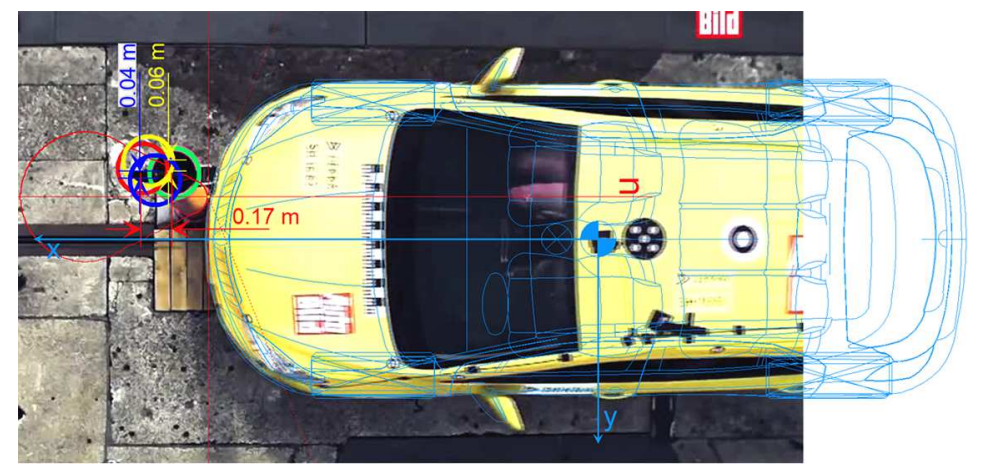

Fig. 8. Deflection of the end of the pillar during the collision for: $t=0.0 \mathrm{~ms}$ - green circle, $t=0.40 \mathrm{~ms}-$ red circle, $t=0.60 \mathrm{~ms}-$ yellow circle, $t=0.80 \mathrm{~ms}-$ blue circle

Next, at $t=60 \mathrm{~ms}$ the engine is turned, the control arm and the half-shaft break off; the pillar penetrates deep into the engine compartment, and hits the bulkhead (Figs. 7b and 9d). At this time, an agreement of the positions is obtained for the right mirror while the lower edge of the windscreen is deformed. The real vehicle moves towards $y$ axis, because the pillar is between the engine and the side member, which causes the aforementioned lateral displacement of the vehicle $(0.20 \mathrm{~m})$. At $t=60 \mathrm{~ms}$, the pillar is deflected by $0.06 \mathrm{~m}$ in the $-y$ direction, whereas at $t=80 \mathrm{~ms}$ the pillar is deflected by $0.04 \mathrm{~m}$ towards the $y$ axis. It shows that the real lateral force acts and changes the direction. This phenomenon is not reflected by the MBS program. The magnitude of the modelled contact force stabilises near $380 \mathrm{kN}$, which is the so-called saturation force (Fig. 10a). Deflection of the pillar towards $x$ axis is similar at $t=40,60$ and $80 \mathrm{~ms}$, which leads to the conclusion that the magnitude of the real contact force can be stable within this time interval (Fig. 8). 

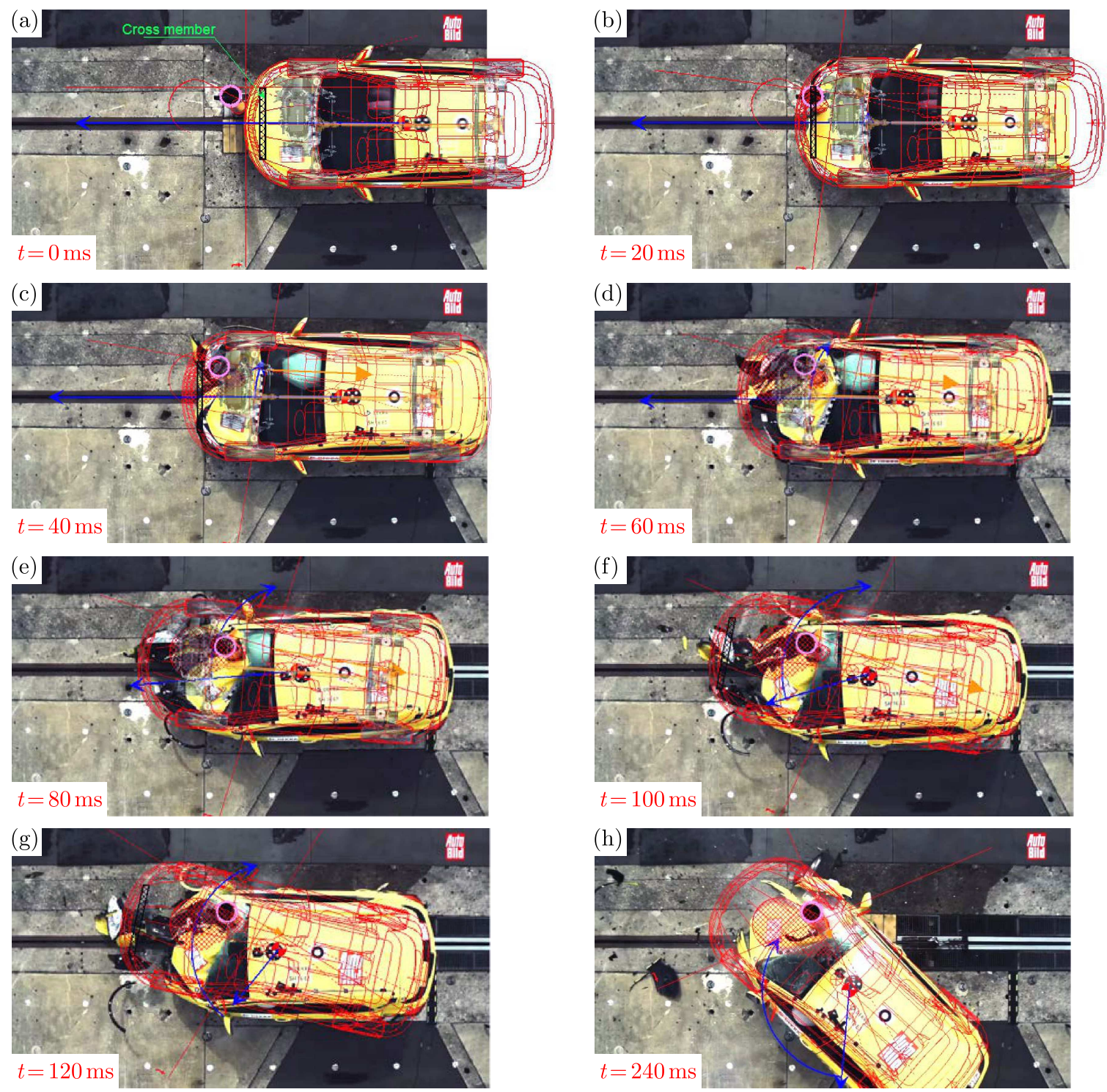

Fig. 9. Comparison of the experimental results with the results of simulation obtained for the identified parameters

In the time interval from 80 to $120 \mathrm{~ms}$, the pillar destroys the safety cage, deforms the floorpan, penetrates the passenger compartment and, consequently, relocates parts from the engine compartment to the passenger compartment. The parts are very near the front of the passenger seat (Fig. 6c). An agreement of the positions is obtained in this case for the upper edge of the windscreen being near the pillar, which in turn shows an agreement of penetrations. Unfortunately, the modelled vehicle turns too early, and the difference between the yaw angles increases with time (Figs. 9e,f,g). The observed differences are mainly a result of the simplified model of contact. As one can see, the MBS program reconstructs the course of the accident with an error.

Finally, at $t=240 \mathrm{~ms}$, one more time a good agreement between the experimental and computational results is obtained (yaw angle and penetration, Fig. 9h). As a result, the upper edges of the windscreen overlap. Summarising, for the modified parameters, a satisfactory agreement between the experimental and computational results is observed. To better illustrate the presented results, a film has been prepared (https://youtu.be/GGCgiY5BMxs). The accident analysis is limited to $t=240 \mathrm{~ms}$, because post-impact movement is limited by tether. 
(a)

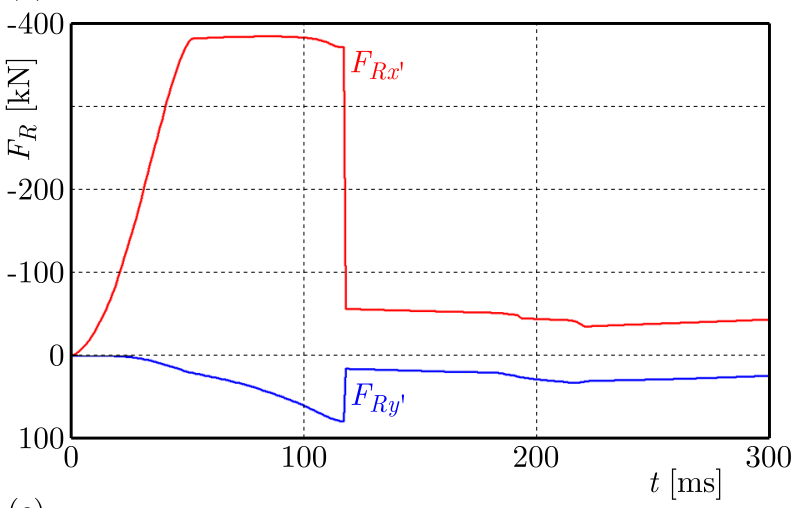

(c)

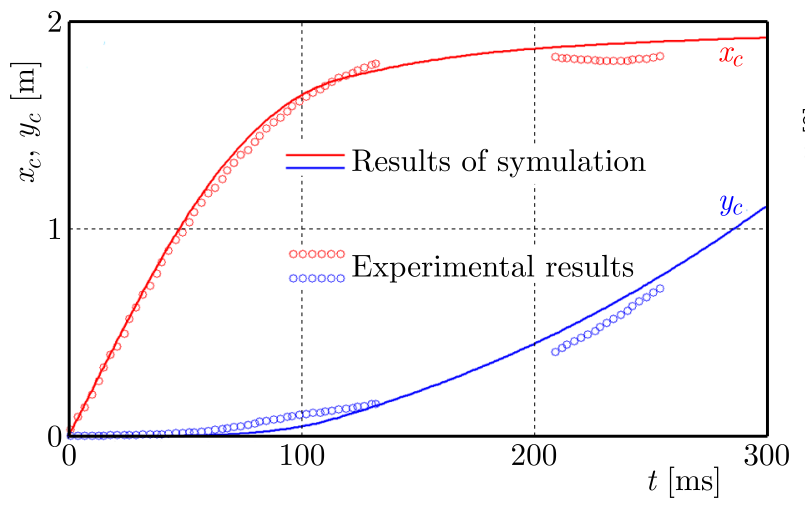

(b)

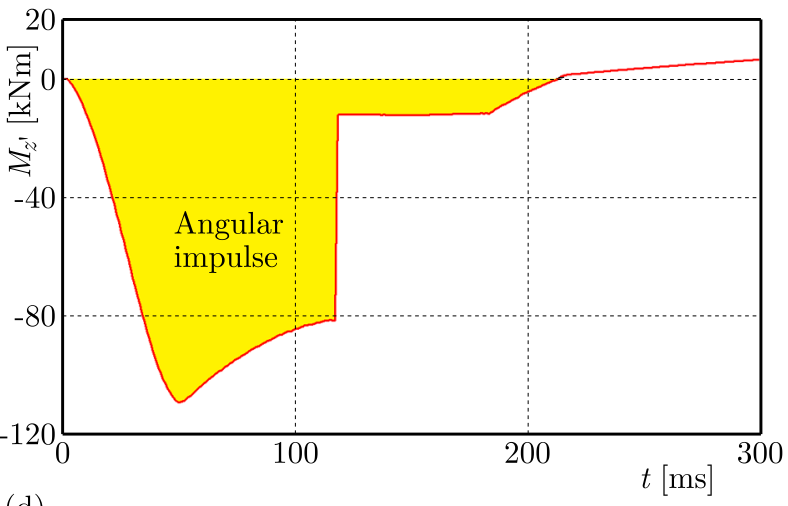

(d)

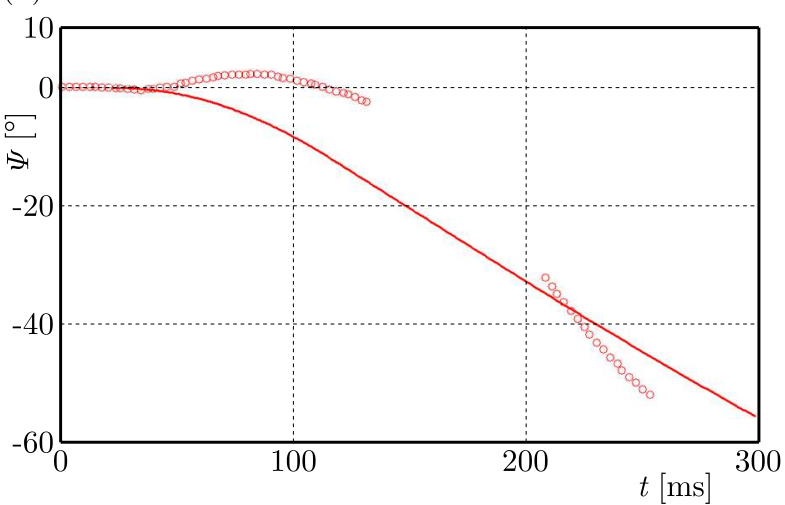

Fig. 10. Time histories of projections of the resultant force $F_{R x^{\prime}} F_{R y^{\prime}}$ acting on the vehicle (a), displacements $x_{c}, y_{c}$ of the centre of mass $C$ in the global system (c), component of the resultant moment acting on the vehicle $M z^{\prime}$ (b), yaw angle $\Psi$ (d)

The time histories of forces, the yaw moment and the displacements are presented in Fig. 10. The magnitude of the component of the resultant force $F_{R x^{\prime}}$ at the initial phase of collision $(0-50 \mathrm{~ms})$ increases, and then is near $380 \mathrm{kN}$ (Fig. 10a). Next, after $t=117 \mathrm{~ms}, F_{R x^{\prime}}$ drops sharply, which corresponds to the end of the compression phase. This drop is observed as well in the second component of the resultant force $F_{R y^{\prime}}$. The acting forces change the motion of the vehicle, which is depicted in Fig. 10c. The vehicle slows down towards the $x$ axis, thus the magnitude of $d x / d t$ drops. Moreover, the vehicle accelerates towards the $y$ axis, and thus gets a certain speed $d y / d t$. Interestingly, a satisfactory agreement between the simulation and experimental results is obtained. Now, the angular motion is described. The angular impulse causing the yaw motion is depicted in Fig. 10b. At the time instant $t=117 \mathrm{~ms}$, the yaw moment $M_{z^{\prime}}$ drops sharply. Next, after $t=216 \mathrm{~ms}$, the yaw speed $d \Psi / d t$ drops. The calculated yaw angle does not show a good agreement with the experimental results, because the MBS program does not model the stiff structures and parts being inside the engine compartment. Modelling these parts is a task for software engineers.

Summarising, many values of parameters were significantly changed to obtain a satisfactory agreement between computational and experimental results. It refers, for example, to the stiffness of the vehicle body and the position of application of the contact force. This leads to the conclusion that this type of crash requires further study and a new model of contact. The program does not automatically introduces the described changes, and does not even suggests introducing any changes. Adopted values of the parameters must be identified experimentally. Moreover, the authors noted that similar results are obtained for a certain range of the parameters. Keeping this in mind, an expert cannot uncritically assume the default data, and indicate guilt based on a single simulation. The sensitivity of a simulated collision pattern to initial conditions was investigated by Wach and Unarski (2007a,b). 


\section{Conclusions}

The values of the parameters assumed in the simulation must be identified from filmed crash tests and real accidents, because for default parameters it is impossible to reconstruct well the course of the crash. This is mostly due to:

- making use of a simplified model of contact between the vehicle and pillar,

- neglecting the internal vehicle structure in the modelling,

- necessity of changing the position of the contact force application point,

- incorrect values of default parameters for this type of crash,

- no automatic decrease of the moments of inertia,

- no automatic blocking of the wheels, and introducing changes to other parameters,

- sensitivity of output data to input data, especially observed in non-linear systems.

Thus, accident reconstruction with MBS programs requires skilled and experienced experts who have to introduce changes to so many parameters; which was surprising. Otherwise, incorrect results of simulation can lead to miscarriages of justice. Despite introducing these many changes, it is impossible to obtain an excellent agreement between time histories. MBS programs do not reflect well severe collisions; they should be rather used to simulate parking fails. Severe collisions should be simulated with FEM programs. Then, deformed parts can be modeled with FEM primitives, whereas undeformed with rigid bodies (primitives); which reduces SPU time. The authors noted that the contact model as well as the $3 \mathrm{D}$ visualisation requires further improvements. 3D visualization provides an opportunity to reduce the parallax error.

\section{References}

1. Abdel-Nasser Y.A., 2013, Frontal crash simulation of vehicles against lighting columns using FEM, Alexandria Engineering Journal, 52, 3, 295-299, DOI: 10.1016/j.aej.2013.01.005

2. AleKsandrowicz P., 2017, Verifying the application of the models of crash and collision detection of incompatible vehicles, 23rd International Conference Engineering Mechanics, Svratka, Czech Republic, 82-85

3. Elmarakbi A., Sennah K., Samann M., Siriya P., 2006, Crashworthiness of motor vehicle and traffic light pole in frontal collisions, Journal of Transportation Engineering (ASCE), 132, 9, 722-733, DOI: 10.1061/(ASCE)0733-947(2006)132:9(722)

4. Ispas N., NAstasoiu M., 2017, Analysis of car's frontal collision against pole, 11th International Congress of Automotive and Transport Engineering, Pitesti, Romania, 1-6, DOI: 10.1088/1757899X/252/1/012012

5. Kostek R., Aleksandrowicz P., 2017a, Simulation of car collision with an impact block, 11th International Congress of Automotive and Transport Engineering, Pitesti, Romania, 1-6, DOI: 10.1088/1757-899X/252/1/012008

6. Kostek R., Aleksandrowicz P., 2017b, Simulation of the right-angle car collision based on identified parameters, 11th International Congress of Automotive and Transport Engineering, Pitesti, Romania, 1-6, DOI: 10.1088/1757-899X/252/1/012013

7. Mnyazikwiye B.B., Karimi H.R., Robbersmyr K.G., 2013, Mathematical modeling of vehicle frontal crash by a double spring-mass-damper model, 24th International Conference on Information, Communication and Automation Technologies (ICAT), Sarajevo, Bosnia and Herzegovina, 1-6, DOI: 10.1109/ICAT.2013.6684071

8. Pawlak M., 2016, The Acceleration Severity Index in the impact of a vehicle against permanent road equipment support structures, Mechanics Research Communications, 77, 21-28, DOI: 10.1016/j.mechrescom.2016.08.005 10. 
9. Pawlus W., Robbersmyr K.G., Karimi H.R., 2011, Mathematical modeling and parameters estimation of a car crash using data-based regressive model approach, Applied Mathematical Modelling, 35, 10, 5091-5107, DOI: 10.1016/j.apm.2011.04.024

10. Pawlus W., Robbersmyr K.G., Karimi H.R., 2013, Investigation of vehicle crash modeling techniques: theory and application, The International Journal of Advanced Manufacturing Technology, 70, 5-8, 965-993, DOI: 10.1007/s00170-013-5320-3

11. Prochowski L., Żuchowski A., 2006, Dynamic loads of power unit during car impact, Journal of KONES Powertrain and Transport, 13, 4, 467-475

12. Radu A.I., Cofaru C., 2015, Study of current state of crash testing, Series I, Engineering Sciences, Bulletin of the Transilvania University of Brasov, 8, 57, 2, 31-36

13. RILl G., 2007, Simulation von Kraftfahrzeugen, Vieweg-Verlag, Genehmigter Nachdruck

14. RüCKER P., 2016, Hier zerschellt ein 5 - Sterne - Auto, AUTOBILD, 8, 51-53

15. Stopel M., Skibicki D., 2016, Determination of Johnson-Cook model constants by measurement of strain rate by optical method, AIP Conference Proceedings, 1780, 060003

16. WACH W., UNARSKI J., 2007a, Determination of collision location - uncertainty analysis by means of Monte Carlo simulation, 16th EVU Annual Meeting Proceedings, Cracow, 73-93

17. WACH W., UNARSKI J., 2007b, Uncertainty of calculation results in vehicle collision analysis, Forensic Science International, 167, 2-3, 181-188

18. York A.R., DAY T.D., 1999, The DyMesh method for three-dimensional multi-vehicle collision simulation, SAE Paper, 1999-01-0104, DOI: 10.4271/1999-01-0104 